\title{
Dissipative boundary control systems with application to distributed parameters reactors
}

\author{
Y. Le Gorrec , B. Maschke, J.A. Villegas, and H. Zwart
}

\begin{abstract}
In this paper we consider distributed parameter physical systems composed of a reversible part associated with a skew-symmetric operator $\mathcal{J}$ as Hamiltonian systems [5] and a symmetric operator associated with some irreversible phenomena. We will show how to use results obtained on reversible systems to parametrize the boundary conditions such that the solution of the associated PDE is contractive. The theoretical results are applied to the example of a tubular reactor with first order chemical reaction. The obtained parametrization is compared with the classical Dankwert conditions.
\end{abstract}

\section{INTRODUCTION}

In this paper we consider distributed parameter reactor with control and observation through the boundary. More precisely we consider a first order chemical reaction of the form :

$$
C_{1} \rightarrow \nu C_{2},
$$

where $C_{1}$ is the reactant, $C_{2}$ the product and $\nu>0$ is the stoichiometric coefficient of the reaction, in a tubular reactor where convection and dissipation phenomena occur. Using mass balance equation on $C_{1}$ for $t \geq 0$ and for $z \in[a, b]=L$ where $L$ is the length of the tubular reactor and, in a first approximation, that $C_{1}$ and $C_{2}$ are at the thermodynamic equilibrium with mass conservation, we can write the following PDE :

$$
\frac{\partial x_{1}}{\partial t}=D_{a} \frac{\partial^{2} x_{1}}{\partial z^{2}}-v \frac{\partial x_{1}}{\partial z}-r\left(x_{1}, x_{2}\right)
$$

Y. Le Gorrec and B. Maschke are with LAGEP, UCB Lyon 1 - UFR - CNRS UMR 5007, CPE Lyon - Bâtiment 308 G, Université Claude Bernard Lyon-1, 43, bd du 11 Novembre 1918, F-69622 Villeurbanne cedex, France \{legorrec, maschke\}alagep.univ-lyon1.fr

The contribution of Y. Le Gorrec and B. Maschke has been done within the context of the European sponsored project GeoPlex with reference code IST-2001-34166. Further information is available at http://www.geoplex.cc.

J.A. Villegas and H. Zwart are with Department of Applied Mathematics, Faculty of Electrical Engineering, Mathematics and Computer Science, University of Twente, P.O. Box 217, 7500 AE Enschede, The Netherlands

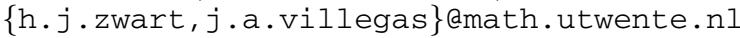

where $x_{1}(z, t)$ is the concentrations of $C_{1}(\mathrm{~mol} / \mathrm{l}), v$ the fluid superficial velocity $(\mathrm{m} / \mathrm{s}), D_{a}$ the axial dispersion coefficient $\left(\mathrm{m}^{2} / \mathrm{s}\right)$ and $r$ the reaction rate. In the following we consider a reaction rate on the form $r=k_{0} x_{1}$ where $k_{0}$ is the kinetic constant $s^{-1}$. Using this hypothesis the model (2) becomes linear :

$$
\frac{\partial x_{1}}{\partial t}=D_{a} \frac{\partial^{2} x_{1}}{\partial z^{2}}-v \frac{\partial x_{1}}{\partial z}-k_{0} x_{1}
$$

The differential operator of such systems can be split into both symmetric and skew symmetric parts and the dynamic equation can be written :

$\frac{\partial x_{1}(t, z)}{\partial t}=\left(\mathcal{J}-\mathcal{G}_{R} S \mathcal{G}_{R}^{*}\right) x_{1}(t, z), x_{1}(0, z)=x_{0,1}(z)$,

where $\mathcal{G}_{R}=\sqrt{D_{a}} \frac{\partial}{\partial z}+\sqrt{k_{0}}, S=I, \mathcal{G}_{R}^{\star}=$ $-\sqrt{D_{a}} \frac{\partial}{\partial z}+\sqrt{k_{0}}$. Associated with this PDE one can define the boundary operators $\mathcal{B}$ and $\mathcal{C}$ as linear operators from $\left\{x \in H^{N}\left((a, b) ; \mathbb{R}^{n}\right) \mid S \mathcal{G}_{R}^{*} x \in\right.$ $\left.H^{N}\left((a, b) ; \mathbb{R}^{n}\right)\right\}$ to $\mathbb{R}^{2 n N}$ only depending on the values at the positions $z=a$ and $z=b$ of $x(t, z)$ and its derivatives up to an order of $N-1$ :

$$
\begin{aligned}
& u(t)=\mathcal{B} x(t, z), \quad z \in(a, b), t \geq 0 \\
& y(t)=\mathcal{C} x(t, z),
\end{aligned}
$$

Under thermodynamic equilibrium hypothesis the modelling of such systems leads to a Sturm-Liouville kind of model. In order to determine a unique solution of this PDE it is necessary to specify the initial condition i.e. $x_{0}(z)$ over the spatial domain and some conditions on the solution at the boundary. Well known boundary conditions, as Dankwert conditions [6], are deduced from physical consideration but usually it is very difficult to parametrize the boundary conditions that ensure the existence of solutions. In this paper we propose to modelize tubular reactor using a Port Based approach. With this kind of approach we'll show that the initial system can be put under the form of a open loop system defined from skew symmetric operator and a coercive feedback. Once this manipulation done, it is possible to use the results stated in [9] to parametrize the boundary conditions that ensure that the initial differential operator defines a contraction semi-group. Roughly speaking, this means that (3)-(5a) has a unique mild 
solution and that the energy of the system is nonincreasing for $u=0$.

\section{INTERCONNECTION STRUCTURE FOR REVERSIBLE AND IRREVERSIBLE SYSTEMS}

We shall consider the systems representing distributed parameter physical systems composed of a reversible part associated with a skew-symmetric operator $\mathcal{J}$ as Hamiltonian systems [5] and a symmetric operator associated with some irreversible phenomena. The irreversible phenomena are described by some constitutive relations defined by the operator $S$ which acts on the state variables via a linear map given by the operator $\mathcal{G}$.

Hence the systems that we consider have the following general form defined by :

$\frac{\partial x}{\partial t}(t, z)=\left(\mathcal{J}-\mathcal{G}_{R} S \mathcal{G}_{R}^{*}\right) x(t, z), x(0, z)=x_{0}(z)$,

where $S$ is a coercive operator on $L_{2}\left((a, b) ; \mathbb{R}^{n}\right)$ and the differential operators $\mathcal{J}$ and $\mathcal{G}_{R}$ are given by

$$
\mathcal{J} x=\sum_{i=0}^{N} P_{i} \frac{\partial^{i} x}{\partial z^{i}}
$$

and

$$
\mathcal{G}_{R} x=\sum_{i=0}^{N} G_{i} \frac{\partial^{i} x}{\partial z^{i}}, \mathcal{G}_{R}^{*} x=\sum_{i=0}^{N}(-1)^{i} G_{i}^{T} \frac{\partial^{i} x}{\partial z^{i}},
$$

with $G_{i}, P_{i}, i=\{1,2, \ldots, N\}$, constant matrices of size $n \times m$, and $n \times n$, respectively. Furthermore, the operator $\mathcal{J}$ is assumed to be formally skewsymmetric which implies that the matrices $P_{i}$ satisfy

$$
P_{i}=(-1)^{i+1} P_{i}^{T}, \quad i=1,2, \ldots, N,
$$

and

$$
\left[\begin{array}{cc}
P_{N} & G_{N} \\
G_{N}^{T} & 0
\end{array}\right] \text { has full rank. }
$$

Here, $\mathcal{G}_{R}^{*}$ is the formal adjoint of $\mathcal{G}_{R}$.

\section{A. Interconnection structure and associated skew- symmetric operators}

In order to deal with systems of the form (4) we consider the higher-dimensional operator:

$$
\mathcal{J}_{e}=\left[\begin{array}{cc}
\mathcal{J} & \mathcal{G}_{R} \\
-\mathcal{G}_{R}^{*} & 0
\end{array}\right]
$$

where $\mathcal{J}, \mathcal{G}_{R}$, and $\mathcal{G}_{R}^{*}$ are given by (7)-(9). This operator makes explicit the interconnection structure associated with the power continuous energy flows in the system defined by the operators $\mathcal{J}$ and $\mathcal{S}$ [7] [4].
By considering the constitutive relations associated with the irreversible phenomena:

$$
e_{p}=S f_{p}
$$

defined by $S$ a coercive operator on $L_{2}\left((a, b) ; \mathbb{R}^{n}\right)$ and the power continuous relations:

$$
\left(\begin{array}{c}
f \\
f_{p}
\end{array}\right)=\mathcal{J}_{e}\left(\begin{array}{c}
e \\
e_{p}
\end{array}\right)=\left[\begin{array}{cc}
\mathcal{J} & \mathcal{G}_{R} \\
-\mathcal{G}_{R}^{*} & 0
\end{array}\right]\left(\begin{array}{c}
e \\
e_{p}
\end{array}\right)
$$

then one recovers the differential operator of the model:

$$
f=\mathcal{J} e-\mathcal{G}_{R} S \mathcal{G}_{R}^{*} e,
$$

which is the same operator that appears in (4). This may be seen as a feedback on the port variables $e_{p}$ and $f_{p}$, feedback will be used to prove the main results in the next section.

The higher-dimensional operator $\mathcal{J}_{e}$ is also formally skew-symmetric, which means that we can use the results given in [9] and [8].

Proposition II.1. The operator $\mathcal{J}_{e}$ defined by (11), (7), and (9) is formally skew-symmetric and can be written as:

$\mathcal{J}_{e}\left(\begin{array}{c}e \\ e_{r}\end{array}\right)=\sum_{i=0}^{N}\left[\begin{array}{cc}P_{i} & G_{i} \\ (-1)^{(i+1)} G_{i}^{T} & 0\end{array}\right] \frac{\partial^{i}}{\partial z^{i}}\left(\begin{array}{c}e \\ e_{r}\end{array}\right)$.

with

$$
\widetilde{P}_{i}
$$

and

$$
\widetilde{P}_{i}=(-1)^{i+1} \widetilde{P}_{i}^{T}
$$

\section{BOUNDARY CONTROL SYSTEMS (BCS) FOR DISSIPATIVE DISTRIBUTED PARAMETER SYSTEMS}

A. Reminder on port variables and boundary control systems associated with skew-symmetric operators

Let us first recall briefly the definition of boundary control systems according to $[1, \S 3.3]$. That is, BCS of the form

$$
\begin{aligned}
\dot{x}(t) & =\mathfrak{A} x(t), \quad x(0)=x_{0}, \\
u(t) & =\mathfrak{B} x(t),
\end{aligned}
$$

where $\mathfrak{A}: D(\mathfrak{A}) \subset X \rightarrow X, u(t) \in U$, a separable Hilbert space, and the boundary operator $\mathfrak{B}: D(\mathfrak{B}) \subset X \rightarrow U$ satisfying $D(\mathfrak{A}) \subset D(\mathfrak{B})$, and

Definition III.1. The control system (15) is a boundary control system if the following hold:

a. The operator $A: D(A) \rightarrow X$ with $D(A)=$ $D(\mathfrak{A}) \cap \operatorname{ker}(\mathfrak{B})$ and

$$
A x=\mathfrak{A} x \quad \text { for } x \in D(A)
$$


is the generator of a $\mathrm{C}_{0}$-semigroup on $X$.

b. There exists a $B \in \mathcal{L}(U, X)$ such that for all $u \in U, B u \in D(\mathfrak{A})$, the operator $\mathfrak{A} B$ is an element of $\mathcal{L}(U, X)$ and $\mathfrak{B} B u=u$ for $u \in U$.

In [9], the authors have parameterized the boundary conditions for which a formally skew-symmetric operator generates a contraction semigroup. We will use their result to proved a similar result for our class of systems. In the remainder of this section we state some results, which are collected from [9].

Theorem III.2. Let $\mathcal{J}_{e}$ be a skew symmetric operator defined by (11), and let $H^{N}\left((a, b) ; \mathbb{R}^{2 n}\right)$ denote the Sobolev space of $N$ times differentiable functions on the interval $(a, b)$. Then for any two functions $e_{e, i}=\left(\begin{array}{c}e_{1} \\ e_{r}\end{array}\right) \in H^{N}\left((a, b) ; \mathbb{R}^{2 n}\right), i \in\{1,2\}$ we have that

$$
\begin{aligned}
& \int_{a}^{b} e_{e, 1}^{T}(z)\left(\mathcal{J}_{e} e_{e, 2}\right)(z)+e_{e, 2}^{T}(z)\left(\mathcal{J}_{e} e_{e, 1}\right)(z) d z= \\
& {\left[\left(\begin{array}{ccc}
e_{e, 1}^{T}(z), & \cdots & \frac{d^{N-1} e_{e, 1}^{T}}{d z^{N-1}}(z)
\end{array}\right) Q\left(\begin{array}{c}
e_{e, 2}(z) \\
\vdots \\
\frac{d^{N-1} e_{e, 2}}{d z^{N-1}}(z)
\end{array}\right)\right]_{(16)}^{b}}
\end{aligned}
$$

where

$Q=\left(\begin{array}{cccccc}\widetilde{P}_{1} & \widetilde{P}_{2} & \widetilde{P}_{3} & \cdots & \widetilde{P}_{N-1} & \widetilde{P}_{N} \\ -\widetilde{P}_{2} & -\widetilde{P}_{3} & -\widetilde{P}_{4} & \cdots & \widetilde{P}_{N} & 0 \\ \vdots & \ddots & \ddots & \ddots & & \vdots \\ (-1)^{N-1} \widetilde{P}_{N} & 0 & \cdots & \cdots & & 0\end{array}\right)_{(17)}$

with $\widetilde{P}_{i}$ given by (13). Furthermore, $Q$ is a nonsingular symmetric matrix.

Definition III.3. The matrix $Q_{\text {ext }}$ in $\mathbb{R}^{4 n N \times 4 n N}$ associated with the differential operator $\mathcal{J}_{e}$ is defined by:

$$
Q_{\mathrm{ext}}=\left(\begin{array}{cc}
Q & 0 \\
0 & -Q
\end{array}\right)
$$

Lemma III.4. The matrix $R_{\mathrm{ext}}$ defined as

$$
R_{\mathrm{ext}}=\frac{1}{\sqrt{2}}\left[\begin{array}{cc}
Q & -Q \\
I & I
\end{array}\right]
$$

is invertible, and satisfies

$$
\left(\begin{array}{cc}
Q & 0 \\
0 & -Q
\end{array}\right)=R_{\text {ext }}^{T} \Sigma R_{\text {ext }}
$$

where

$$
\Sigma=\left(\begin{array}{ll}
0 & I \\
I & 0
\end{array}\right)
$$

All possible matrices $R$ which satisfies (20) are given by the formula

$$
R=U R_{\text {ext }},
$$

with $U$ satisfying $U^{T} \Sigma U=\Sigma$.

Definition III.5. The boundary port variables associated with the differential operator $\mathcal{J}_{e}$ are the vectors $e_{e, \partial}, f_{e, \partial} \in \mathbb{R}^{2 n N}$, defined by

$$
\left(\begin{array}{c}
f_{e, \partial} \\
e_{e, \partial}
\end{array}\right)=R_{\text {ext }}\left(\begin{array}{c}
e(b) \\
e_{r}(b) \\
\vdots \\
\frac{d^{N-1} e}{d z^{N-1}}(b) \\
\frac{d^{N-1} e_{r}}{d z^{N-1}}(b) \\
e(a) \\
e_{r}(a) \\
\vdots \\
\frac{d^{N-1} e}{d z^{N-1}}(a) \\
\frac{d^{N-1} e_{r}}{d z^{N-1}}(a)
\end{array}\right),
$$

where $R_{\text {ext }}$ is defined by (19).

Following Theorem 4.2 of [9] we immediately obtain the following result.

Theorem III.6. Let $W$ be a $2 n N \times 4 n N$ matrix. If $W$ has full rank and satisfies $W \Sigma W^{T} \geq 0$, where $\Sigma$ is defined in (21), then the system

$$
\frac{\partial x}{\partial t}(t)=\mathcal{J}_{e} x(t)
$$

with input

$$
u(t)=W\left(\begin{array}{l}
f_{e, \partial}(t) \\
e_{e, \partial}(t)
\end{array}\right)
$$

is a boundary control system. Furthermore, the operator $A_{\mathrm{ext}}=\mathcal{J}_{e}$ with domain

$D\left(A_{\text {ext }}\right)=\left\{\left(\begin{array}{c}e \\ e_{r}\end{array}\right) \in\left(\begin{array}{c}H^{N}\left((a, b), \mathbb{R}^{n}\right) \\ H^{N}\left((a, b), \mathbb{R}^{n}\right)\end{array}\right) \mid\left(\begin{array}{c}f_{e, \partial} \\ e_{e, \partial}\end{array}\right) \in \operatorname{ker} W\right\}$,

generates a contraction semigroup.

Let $\tilde{W}$ be a full rank matrix of size $2 n N \times 4 n N$ with $\left(\begin{array}{c}W \\ \tilde{W}\end{array}\right)$ invertible. If we define the linear mapping $\mathcal{C}: H^{N}\left((a, b), \mathbb{R}^{2 n}\right) \rightarrow \mathbb{R}^{2 n N}$ as,

$$
\mathcal{C} x(t):=\tilde{W}\left(\begin{array}{l}
f_{e, \partial}(t) \\
e_{e, \partial}(t)
\end{array}\right)
$$

and the output as

$$
y(t)=\mathcal{C} x(t)
$$

then for $u \in C^{2}\left((0, \infty) ; \mathbb{R}^{2 n N}\right), \quad x(0) \in$ $H^{N}\left((a, b), \mathbb{R}^{2 n}\right)$, and $\mathcal{B} x(0)=u(0)$ the following balance equation is satisfied:

$\frac{1}{2} \frac{d}{d t}\|x(t)\|^{2}=\frac{1}{2}\left(\begin{array}{ll}u^{T}(t) & y^{T}(t)\end{array}\right) P_{W, \tilde{W}}\left(\begin{array}{c}u(t) \\ y(t)\end{array}\right)$,

where

$$
P_{W, \tilde{W}}^{-1}=\left[\begin{array}{ll}
W \Sigma W^{T} & W \Sigma \tilde{W}^{T} \\
\tilde{W} \Sigma W^{T} & \tilde{W} \Sigma \tilde{W}^{T}
\end{array}\right]
$$

Furthermore, we have that the matrix $\left(\begin{array}{cc}W \Sigma W^{T} & W \Sigma \tilde{W}^{T} \\ \tilde{W} \Sigma W^{T} & \tilde{W} \Sigma \tilde{W}^{T}\end{array}\right)$ is invertible if and only if $\left(\begin{array}{l}W \\ \tilde{W}\end{array}\right)$ is invertible. 
B. Boundary control system associated with irreversible linear systems

First observe that if we define

$$
\left(\begin{array}{c}
f \\
f_{p}
\end{array}\right)=\mathcal{J}_{e}\left(\begin{array}{c}
e \\
e_{p}
\end{array}\right)=\left[\begin{array}{cc}
\mathcal{J} & \mathcal{G}_{R} \\
-\mathcal{G}_{R}^{*} & 0
\end{array}\right]\left(\begin{array}{c}
e \\
e_{p}
\end{array}\right)
$$

and let $e_{p}=S f_{p}$ with $S$ a coercive operator on $L_{2}\left((a, b) ; \mathbb{R}^{n}\right)$, we obtain

$$
f=\mathcal{J} e-\mathcal{G}_{R} S \mathcal{G}_{R}^{*} e,
$$

which is the same operator that defines our class of systems. This idea of feedback will be used to prove the next theorem. In order to simplify the notation we introduce the following definition.

Definition III.7. The boundary port variables associated with the differential operator $\left(\mathcal{J}-\mathcal{G}_{R} S \mathcal{G}_{R}^{*}\right)$ are the vectors $g_{f, \partial}, g_{e, \partial} \in \mathbb{R}^{2 n N}$, defined by

$$
\left(\begin{array}{c}
g_{f, \partial} \\
g_{e, \partial}
\end{array}\right)=R_{\operatorname{ext}}\left(\begin{array}{c}
e(b) \\
\left(-S \mathcal{G}_{R}^{*} e\right)(b) \\
\vdots \\
\frac{d^{N-1} e}{d z^{N-1}(b)} \\
\left.d z^{N-1} e\right) \\
e(a) \\
\left(-S \mathcal{G}_{R}^{*} e\right)(a) \\
\vdots \\
\frac{d^{N-1}\left(-S \mathcal{G}_{R}^{*} e\right)}{d z^{N-1}}(a)
\end{array}\right)
$$

where $R_{\text {ext }}$ is defined by (19).

Remark III.8. Observe that (28) is the same as (22) whenever $e_{e}=\left(\begin{array}{c}e \\ -S \mathcal{G}_{R}^{*} e\end{array}\right)$.

Theorem III.9. Consider the operator $A=(\mathcal{J}-$ $\left.\mathcal{G}_{R} S \mathcal{G}_{R}^{*}\right)$ with domain

$$
\begin{aligned}
D(A)=\left\{e \in H^{N}\left((a, b) ; \mathbb{R}^{n}\right) \mid\right. & S \mathcal{G}_{R}^{*} e \in H^{N}\left((a, b) ; \mathbb{R}^{n}\right), \\
& \left.\left(\begin{array}{c}
g_{f, \partial} \\
g_{e, \partial}
\end{array}\right) \in \operatorname{ker} W\right\}
\end{aligned}
$$

If $W$ has full rank and satisfies $W \Sigma W^{T} \geq 0$, then A generates a contraction semigroup.

Proof: As mentioned before, the proof is based on a feedback argument on the operator $\mathcal{J}_{e}$. First observe that since $A_{\text {ext }}$ is the generator of a contraction semigroup (see Theorem III.6) we have from the Lümer-Phillips theorem (see [3, Theorem 2.27]) that

$\left\langle A_{\text {ext }} \tilde{e}, \tilde{e}\right\rangle \leq 0$ for all $\tilde{e} \in D\left(A_{\text {ext }}\right)$ and $\operatorname{ran}\left(\lambda I-A_{\text {ext }}\right)=L_{2}\left((a, b) ; \mathbb{R}^{2 n}\right)$ for some $\lambda>0$.

Now we can proceed to prove that $A$ generates a contraction semigroup. To do so, we will use the same Lümer-Phillips theorem. That is, we first prove that $A$ satisfies $\langle A e, e\rangle \leq 0$ for any $e \in D(A)$ and next that $\operatorname{ran}(\lambda I-A)=L_{2}\left((a, b) ; \mathbb{R}^{n}\right)$ for some $\lambda>0$. For $e \in D(A)$, we have

$\langle A e, e\rangle=\left\langle\left(\mathcal{J}-\mathcal{G}_{R} S \mathcal{G}_{R}^{*}\right) e, e\right\rangle=\langle\mathcal{J}, e\rangle+\left\langle-\mathcal{G}_{R} S \mathcal{G}_{R}^{*} e, e\right\rangle$.

Define $e_{p}=-S \mathcal{G}_{R}^{*} e$ and observe that $e_{p} \in$ $H^{N}\left((a, b) ; \mathbb{R}^{n}\right)$, see (29). It is now easy to see that $\left(\begin{array}{c}e \\ e_{p}\end{array}\right) \in D\left(A_{\text {ext }}\right)$, see Remark III.8 and (23). From this and the equation above we can see that

$$
\begin{aligned}
\langle A e, e\rangle & =\left\langle\mathcal{J} e+\mathcal{G}_{R} e_{p}, e\right\rangle \\
& \leq\left\langle\mathcal{J} e+\mathcal{G}_{R} e_{p}, e\right\rangle+\left\langle\mathcal{G}_{R}^{*} e, S \mathcal{G}_{R}^{*} e\right\rangle \\
& =\left\langle\mathcal{J} e+\mathcal{G}_{R} e_{p}, e\right\rangle+\left\langle\mathcal{G}_{R}^{*} e,-e_{p}\right\rangle \\
& =\left\langle\left[\begin{array}{cc}
\mathcal{J} & \mathcal{G}_{R} \\
-\mathcal{G}_{R}^{*} & 0
\end{array}\right]\left(\begin{array}{c}
e \\
e_{p}
\end{array}\right),\left(\begin{array}{c}
e \\
e_{p}
\end{array}\right)\right\rangle \\
& =\left\langle A_{\operatorname{ext}}\left(\begin{array}{c}
e \\
e_{p}
\end{array}\right),\left(\begin{array}{c}
e \\
e_{p}
\end{array}\right)\right\rangle \leq 0,
\end{aligned}
$$

where in the second step we used the fact that $S$ is coercive, in the third step we used $e_{p}=-S \mathcal{G}_{R}^{*} e$, and in the last step we used (31).

Next we prove the range condition on $A$. That is, for a $\lambda>0$ we have to prove that for any given $f \in L_{2}\left((a, b) ; \mathbb{R}^{n}\right)$ we can find an $e \in D(A)$ such that

$$
f=(\lambda I-A) e .
$$

In order to prove this, let

$$
P=\left[\begin{array}{cc}
0 & 0 \\
0 & S^{-1}-\lambda I
\end{array}\right] \text {. }
$$

Since $S$ is coercive, we can find some $\lambda>0$ such that $S^{-1}-\lambda I \geq 0$. Thus we can assume that $P$ is a nonnegative operator. Let consider the following technical Lemma :

Lemma III.10. Let $P$ be a nonnegative bounded operator and let $M$ be the generator of a contraction semigroup. Then, $M-P$ also generates a contraction semigroup.

It follows from Lemma III.10 that $A_{\text {ext }}-P$ generates a contraction semigroup. This in turn implies that $\operatorname{ran}\left(\lambda I-A_{\text {ext }}+P\right)=L_{2}\left((a, b) ; \mathbb{R}^{2 n}\right)$. Thus, given any $\left(\begin{array}{l}f \\ 0\end{array}\right) \in L_{2}\left((a, b) ; \mathbb{R}^{2 n}\right)$ we can find $\left(\begin{array}{c}e \\ e_{p}\end{array}\right) \in$ $D\left(A_{\text {ext }}\right)$ such that

$$
\begin{aligned}
& \left(\begin{array}{c}
f \\
0
\end{array}\right)=\left(\lambda I-A_{\text {ext }}+P\right)\left(\begin{array}{c}
e \\
e_{p}
\end{array}\right) \\
= & {\left[\begin{array}{cc}
\lambda I-\mathcal{J} & -\mathcal{G}_{R} \\
\mathcal{G}_{R}^{*} & S^{-1}
\end{array}\right]\left(\begin{array}{c}
e \\
e_{p}
\end{array}\right) } \\
\Rightarrow \quad & f=(\lambda I-\mathcal{J}) e-\mathcal{G}_{R} e_{p} \quad \text { and } \\
& e_{p}=-S \mathcal{G}_{R}^{*} e \\
\Rightarrow \quad & f=\left[\lambda I-\left(\mathcal{J}-\mathcal{G}_{R} S \mathcal{G}_{R}^{*}\right)\right] e .
\end{aligned}
$$

Since $\left(\begin{array}{c}e \\ e_{p}\end{array}\right)=\left(\begin{array}{c}e \\ -S \mathcal{G}_{R}^{*} e\end{array}\right) \in D\left(A_{\text {ext }}\right)$, it is easy to see that $e \in D(A)$. Then from (33) we can see that $A$ 
satisfies the range condition. Concluding, we see that $A$ generates a contraction semigroup.

Following Theorem III.6 and Theorem III.9 we can prove the following result.

Theorem III.11. Let $W$ be a $2 n N \times 4 n N$ matrix. If $W$ has full rank and satisfies $W \Sigma W^{T} \geq 0$, where $\Sigma$ is defined in (21), then the system

$$
\frac{\partial x}{\partial t}(t)=\left(\mathcal{J}-\mathcal{G}_{R} S \mathcal{G}_{R}^{*}\right) x(t)
$$

with input

$$
u(t)=\mathcal{B} x(t)=W\left(\begin{array}{l}
g_{f, \partial}(t) \\
g_{e, \partial}(t)
\end{array}\right)
$$

is a boundary control system. Furthermore, the operator $A=\mathcal{J}-\mathcal{G}_{R} S \mathcal{G}_{R}^{*}$ with domain

$$
\begin{array}{r}
D(A)=\left\{e \in H^{N}\left((a, b) ; \mathbb{R}^{n}\right) \mid S \mathcal{G}_{R}^{*} e \in H^{N}\left((a, b) ; \mathbb{R}^{n}\right),\right. \\
\left.\left(\begin{array}{c}
g_{f, \partial} \\
g_{e, \partial}
\end{array}\right) \in \operatorname{ker} W\right\} .
\end{array}
$$

generates a contraction semigroup.

Let $\tilde{W}$ be a full rank matrix of size $2 n N \times 4 n N$ with $\left(\begin{array}{c}W \\ \tilde{W}\end{array}\right)$ invertible. If we define the linear mapping $\mathcal{C}: H^{N}\left((a, b), \mathbb{R}^{2 n}\right) \rightarrow \mathbb{R}^{2 n N}$ as,

$$
\mathcal{C} x(t):=\tilde{W}\left(\begin{array}{l}
g_{f, \partial}(t) \\
g_{e, \partial}(t)
\end{array}\right)
$$

and the output as

$$
y(t)=\mathcal{C} x(t),
$$

then for $u \in C^{2}\left((0, \infty) ; \mathbb{R}^{2 n N}\right), \quad x(0) \quad \in$ $H^{N}\left((a, b), \mathbb{R}^{2 n}\right)$, and $\mathcal{B} x(0)=u(0)$ the following balance equation is satisfied:

$\frac{1}{2} \frac{d}{d t}\|x(t)\|^{2} \leq \frac{1}{2}\left(\begin{array}{ll}u^{T}(t) & y^{T}(t)\end{array}\right) P_{W, \tilde{W}}\left(\begin{array}{c}u(t) \\ y(t)\end{array}\right)$

where

$$
P_{W, \tilde{W}}^{-1}=\left[\begin{array}{cc}
W \Sigma W^{T} & W \Sigma \tilde{W}^{T} \\
\tilde{W} \Sigma W^{T} & \tilde{W} \Sigma \tilde{W}^{T}
\end{array}\right] .
$$

Furthermore, we have that the matrix $\left(\begin{array}{ll}W \Sigma W^{T} & W \Sigma \tilde{W}^{T} \\ \tilde{W} \Sigma W^{T} & \tilde{W} \Sigma \tilde{W}^{T}\end{array}\right)$ is invertible if and only if $\left(\begin{array}{c}W \\ \tilde{W}\end{array}\right)$ is invertible.

Proof: We divide the proof in three steps. In Step 1. and 2. we show that we have a boundary control system. In step 3. we prove (41) and (42), respectively. For a boundary control system we have to show that for zero inputs, the operator $A$ generates a $C_{0}$-semigroup, and furthermore that there exists a bounded operator $B$ mapping into the domain of $\mathcal{B}$ and such that $\mathcal{B B} u=u$ for all $u \in \mathbb{R}^{2 n N}$.

Step 1: As mentioned above, we have to show that $A=\mathcal{J}-\mathcal{G}_{R} S \mathcal{G}_{R}^{*}$ with domain (37) is an infinitesimal generator of a semigroup. This follows directly from Theorem III.9.

Step 2: We have to find a bounded linear operator $B$ such that $B u \in D(\mathcal{B})=H^{N}\left((a, b) ; \mathbb{R}^{n N}\right)$ and $\mathcal{B} B u=u$ for all $u \in \mathbb{R}^{2 n N}$. This follows similarly as the second step in the proof of Theorem 4.5 of [2].

Step 3: By the definition of $B$ and $D(A)$, we see that the conditions stated in the theorem are the same as $x(0)-B u(0) \in D(A)$. Hence by Theorem 3.3 .3 of [1] we have that there exists a classical solution of (35)-(36). Hence, in particular, $x(t) \in H^{N}\left((a, b), \mathbb{R}^{n}\right)$ holds pointwise in $t, x(t)$ is differentiable as a function of $t$, and $\dot{x}(t)=$ $\left(\mathcal{J}-\mathcal{G}_{R} S \mathcal{G}_{R}^{*}\right) x(t)$. Using this, we obtain

$$
\begin{aligned}
\frac{d}{d t}\|x(t)\|^{2} & =\frac{d}{d t}\langle x(t), x(t)\rangle \\
& =\langle\dot{x}(t), x(t)\rangle+\langle x(t), \dot{x}(t)\rangle \\
& =\left\langle\left(\mathcal{J}-\mathcal{G}_{R} S \mathcal{G}_{R}^{*}\right) x(t), x(t)\right\rangle+\left\langle x(t),\left(\mathcal{J}-\mathcal{G}_{R} S \mathcal{G}_{R}^{*}\right) x(t)\right\rangle
\end{aligned}
$$

Define $x_{p}=-S \mathcal{G}_{R}^{*} x$ and observe that $x_{p} \in$ $H^{N}\left((a, b) ; \mathbb{R}^{n}\right)$, see (37). It is now easy to see that $\left(\begin{array}{c}x \\ x_{p}\end{array}\right) \in D\left(A_{\text {ext }}\right)$, see Remark III.8. From this and the equation above we can see after some calculation that

$$
\begin{aligned}
\frac{d}{d t}\|x(t)\|^{2} & =\left\langle\mathcal{J} x(t)+\mathcal{G}_{R} x_{p}(t), x(t)\right\rangle+\left\langle x(t), \mathcal{J} x(t)+\mathcal{G}_{R} x_{p}(t)\right\rangle \\
& \leq\left\langle\mathcal{J} x(t)+\mathcal{G}_{R} x_{p}(t), x(t)\right\rangle+\left\langle x(t), \mathcal{J} x(t)+\mathcal{G}_{R} x_{p}(t)\right\rangle \\
& =\frac{d}{d t}\left\|\left(\begin{array}{c}
x(t) \\
x_{p}(t)
\end{array}\right)\right\|^{2},
\end{aligned}
$$

where we used the fact that $S$ is coercive, and $x_{p}=-S \mathcal{G}_{R}^{*} x$. The rest of the proof follows from Equations (26) and (27) and the fact that $\left(\begin{array}{c}x \\ x_{p}\end{array}\right) \in$ $D\left(A_{\text {ext }}\right)$.

Remark III.12. Following Section 5 of [9] we can easily see that Theorem III.11 also holds if we replace the operator $\left(\mathcal{J}-\mathcal{G}_{R} S \mathcal{G}_{R}^{*}\right)$ by $\left(\mathcal{J}-\mathcal{G}_{R} S \mathcal{G}_{R}^{*}\right) \mathcal{L}$, where $\mathcal{L}$ is a coercive operator on $L_{2}\left((a, b) ; \mathbb{R}^{n}\right)$. This allows to deal with systems with different parameters or even systems with nonconstant parameters.

C. Boundary control system associated with distributed parameter reactor

Let recall our example of tubular reactor. The considered PDE is

$$
\frac{\partial x_{1}}{\partial t}=D_{a} \frac{\partial^{2} x_{1}}{\partial z^{2}}-v \frac{\partial x_{1}}{\partial z}-k_{0} x_{1}
$$

where $v>0$ is the velocity of the fluid, $D_{a}>0$ the diffusion constant, $k_{0}$ is the kinetic constant. Comparing the equation above with (4), we can easily see that in this case we have

$$
\begin{aligned}
\mathcal{J} & =-v \frac{\partial}{\partial z} \\
-\mathcal{G}_{R} S \mathcal{G}_{R}^{*} & =D_{a} \frac{\partial^{2}}{\partial z^{2}}-k_{0} .
\end{aligned}
$$


From this we get

$$
\mathcal{G}_{R}=\sqrt{D_{a}} \frac{\partial}{\partial z}+\sqrt{k_{0}}, \quad S=I
$$

and

$$
\mathcal{G}_{R}^{*}=-\sqrt{D_{a}} \frac{\partial}{\partial z}+\sqrt{k_{0}},
$$

and thus (see equations (7) and (13)) $N=1$,

$$
P_{1}=-v, G_{1}=\sqrt{D_{a}}, P_{0}=0, G_{0}=\sqrt{k_{0}},
$$

and

$$
\widetilde{P}_{1}=\left[\begin{array}{cc}
-v & \sqrt{D_{a}} \\
\sqrt{D_{a}} & 0
\end{array}\right]=Q
$$

Recall that $\mathcal{G}_{R}^{*}$ is the formal adjoint of $\mathcal{G}_{R}$, i.e. the adjoint of $\mathcal{G}_{R}$ ignoring boundary variables. Then it is easy to see that equation (43) becomes

$$
\frac{\partial C}{\partial t}(t, z)=\left(\mathcal{J}-\mathcal{G}_{R} S \mathcal{G}_{R}^{*}\right) C(t, z)
$$

From Definition III.7 and using (19) we obtain the boundary port variables

$$
\left(\begin{array}{c}
g_{f, \partial} \\
g_{e, \partial}
\end{array}\right)=R_{e x t}\left(\begin{array}{c}
C(b) \\
\sqrt{D_{a}} \frac{\partial C}{\partial z}(b)-\sqrt{k_{0}} C(b) \\
C(a) \\
\sqrt{D_{a}} \frac{\partial C}{\partial z}(a)-\sqrt{k_{0}} C(a)
\end{array}\right)
$$

with

$$
R_{e x t}=\frac{1}{\sqrt{2}}\left(\begin{array}{cccc}
-v & \sqrt{D_{a}} & v & -\sqrt{D_{a}} \\
\sqrt{D_{a}} & 0 & -\sqrt{D_{a}} & 0 \\
1 & 0 & 1 & 0 \\
0 & 1 & 0 & 1
\end{array}\right)
$$

\section{REFERENCES}

[1] R.F. Curtain and H.J. Zwart. An introduction to InfiniteDimensional Linear System Theory. Springer-Verlag, i edition, 1995. ISBN 0-387-94475-3.

[2] Y. Le Gorrec, H. Zwart, and B.M. Maschke. Dirac structures and boundary control systems associated with skewsymmetric differential operators. (Memorandum Faculteit TW no. 1730). Enschede: Universiteit Twente, 32 pp. ISBN 0169 2690.http://www.math.utwente.nl/ssb/annrep04/publs04.htm, 2004.

[3] Z.H. Luo, B.Z. Guo, and O. Morgul. Stability and Stabilization of Infinite Dimensional Systems with Applications. Number ISBN 1-85233-124-0. Springer.

[4] B. Maschke and A.J. van der Schaft. Advanced Topics in Control Systems Theory. Lecture Notes from FAP 2004, chapter Compositional modelling of distributed-parameter systems, pages 115-154. Lecture Notes on Control and Information Sciences. Springer, 2005.

[5] Peter J. Olver. Applications of Lie Groups to Differential Equations, volume 107 of Graduate texts in mathematics. Springer, New-York, ii edition, 1993. ISBN 0-387-94007-3.

[6] D.M. Ruthven. Principles of Adsorption and Adsorption Processes. John Wiley and Sons, New York, 1984.

[7] A.J. van der Schaft and B.M. Maschke. Hamiltonian formulation of distributed parameter systems with boundary energy flow. J. of Geometry and Physics, 42:166-174, 2002.

[8] H. Zwart Y. Le Gorrec and B.M. Maschke. Dirac structures and boundary control systems associated with skewsymmetric differential operators. Memorandum n1730 of the University of Twente, The Netherlands, 2004.

[9] H. Zwart Y. Le Gorrec and B.M. Maschke. Dirac structures and boundary control systems associated with skewsymmetric differential operators. SIAM J. of Control and Optimization, 44(5):1864-1892, 2005.

i.e.

$\left(\begin{array}{c}g_{f, \partial} \\ g_{e, \partial}\end{array}\right)=\frac{1}{\sqrt{2}}\left(\begin{array}{c}-v(C(b)-C(a))+D_{a}\left(\frac{\partial C}{\partial z}(b)-\frac{\partial C}{\partial z}(a)\right)-\sqrt{k_{0} D_{a}}(C(b)-C(a)) \\ \sqrt{D_{a}}(C(b)-C(a)) \\ C(b)+C(a) \\ \sqrt{D_{a}}\left(\frac{\partial C}{\partial z}(b)+\frac{\partial C}{\partial z}(a)\right)-\sqrt{k_{0}}(C(b)+C(a)) \\ (50)\end{array}\right)$

As stated into the introduction, the boundary conditions are usually chosen as Dankwert conditions. This means that the system input is chosen as being the reactant flux at the input $\left(v C(t, a)-D_{a} \frac{\partial C}{\partial z}(t, a)\right)$ of the reactor and that the gradient of the concentration $\left(D_{a} \frac{\partial C}{\partial z}(t, b)\right)$ is assigned to 0 at the output of the reactor. Say, we want

$D_{a} \frac{\partial C}{\partial z}(t, a)-v C(t, a)=v C_{\mathrm{in}}(t), \quad$ and $\quad D_{a} \frac{\partial C}{\partial z}(t, b)=0$,

where $C_{\mathrm{in}}$ is an input function. It is easy to see that these boundary conditions can be obtained from the port variables by premultiplying them by the following matrix

$$
W=\frac{1}{\sqrt{2}}\left(\begin{array}{cccc}
-1 & \frac{v}{2 \sqrt{D_{a}}} & -\frac{v}{2} & \sqrt{D_{a}} \\
1 & \frac{v+\sqrt{k_{0} D_{a}}}{\sqrt{D_{a}}} & \sqrt{k_{0} D_{a}} & \sqrt{D_{a}}
\end{array}\right) .
$$

Since this matrix satisfies $W \Sigma W^{T}=\left[\begin{array}{ll}v & v \\ v & v\end{array}\right] \geq 0$, we have that the results in this section apply to this system. 